\title{
Localisation of Multiple Non-Isolated Microbubbles with Frequency Decomposition in Super-Resolution Imaging
}

\author{
Sevan Harput ${ }^{1}$, Kirsten Christensen-Jeffries ${ }^{2}$, Jemma Brown ${ }^{2}$, \\ Robert J. Eckersley ${ }^{2 *}$, Chris Dunsby ${ }^{3 *}$, and Meng-Xing Tang ${ }^{1 *}$ \\ ${ }^{1}$ ULIS Group, Department of Bioengineering, Imperial College London, London, SW7 2BP, UK \\ ${ }^{2}$ Biomedical Engineering Department, Division of Imaging Sciences, King's College London, SE1 7EH, London, UK \\ ${ }^{3}$ Department of Physics and the Centre for Pathology, Imperial College London, London, SW7 2AZ, UK \\ ${ }^{*}$ These authors contributed equally to this work \\ E-mail: S.Harput@imperial.ac.uk, Mengxing.Tang@imperial.ac.uk
}

\begin{abstract}
Sub-diffraction imaging, also known as ultrasound localization microscopy, is a novel method that can overcome the fundamental diffraction limit by localizing spatially isolated microbubbles. This method requires the use of a low concentration of microbubbles to ensure that they are spatially isolated. For in vivo microvascular imaging, especially for cancer tissue with high microvascular density, spatial isolation cannot be always achieved, since vessels are close to each other and the speed of flow is slow.

This study proposes a frequency decomposition method that uses the polydisperse nature of commercial contrast agents to separate spatially non-isolated microbubbles with different acoustic signatures. Zero-phase filters were applied to ensure that there is no relative phase delay between decomposed signals. Results showed that a super-resolution image after frequency decomposition can be generated with 1.4 times lower number of acquisitions.
\end{abstract}

\section{INTRODUCTION}

Photo activated localization microscopy (PALM) and stochastic optical reconstruction microscopy (STORM) use photo-switchable dyes and protein fluorophores to achieve super-resolution images by activating and localizing only an optically resolvable subset of these agents. These stochastic localisation microscopy techniques rely on the detection of spatially isolated fluorophores at any given frame so that their position can be measured with high precision. Currently, most of the ultrasound localisation microscopy techniques use the same idea to form a super-resolution image by localizing spatially isolated microbubbles through multiple frames. Within last five years, several research groups demonstrated the use of this super-resolution method within microfluidic channels, tissue phantoms with microvessels, through an ex vivo human skull model, and pre-clinical mouse models [1]-[6]. Microbubble localization precision as small as $2-4 \mu \mathrm{m}$ was reported using clinically relevant ultrasound frequencies [1], [7]. These methods require 10-20 minutes of ultrasound acquisition to visualise the microvascular structures due to the slow flow rate in capillaries.
Super-resolution optical fluctuation imaging (SOFI) uses the higher-order cumulants to produce super-resolution images. By generating virtual pixels based on the temporal autocorrelation of pixels, this method can achieve a more precise localisation than the aforementioned methods when the density of activated fluorophores is too high. It is also possible to use a similar approach and a high concentration of microbubbles to form ultrasound super-resolution images. Bar-Zion et al. proposed a model utilizing higher order statistics by acquiring less than a second of high frame rate ultrasound data. They achieved a modest improvement of 50\% in spatial resolution with a significantly shorter acquisition time by localization of spatially non-isolated microbubbles [8]. Although this method is more practical, the final resolution of the super-resolution images is not as impressive as methods based on localizing spatially isolated microbubbles.

A low concentration of microbubbles is required to achieve isolated signals in microvasculature, which is especially challenging in cancerous tissue due to high vessel density and slow flow. Therefore, generating a super-resolution image requires a longer acquisition time to cover the same region with spatially isolated microbubbles. In this study, a zero-phase filtering method is used to decompose the microbubble signal into different frequency components. The proposed method takes advantage of the polydisperse nature of commercial microbubble contrast agents with different acoustic signatures to separate spatially non-isolated microbubble signals.

\section{Materials AND Methods}

\section{A. Frequency Decomposition}

This study utilizes zero-phase filtering for signal decomposition in the frequency domain. Zero-phase filtering was chosen due to the ease of implementation and computational speed.

Conventional methods used to decompose a signal into multiple frequency components introduce phase delays between components. After filtering or decomposing the signal into multiple components different phase shifts might be observed 
between the components with different frequency content. For example, FIR filters have linear phase response. This means the phase delay between the frequency components are changing linearly. For conventional ultrasound imaging this is negligible since reflections from tissue almost have same frequency content. After FIR filtering the echoes from different parts of tissue experiences the same phase shift, which introduces a bias instead of an error. However, when localising microbubbles to below the diffraction limit, even a FIR filter with a linear phase response will introduce a different phase shift between different microbubble signals since they may have a different spectral content.

Therefore, multiple zero-phase filters were implemented by bandpass filtering the signal twice and performing a time reversal operation after each filtering stage. Since time reversal operation in time domain corresponds to complex conjugate in frequency domain, the phase information of the filter is cancelled. This procedure cancels the unwanted phase shifts and hence the time delay between decomposed signals. While imaging the microvasculature, a $40 \mathrm{~ns}$ shift between decomposed signals can cause up to $30 \mu \mathrm{m}$ error in the final superresolution image.

Zero-phase filtering is four stage operation, where a signal $x(t)$ is filtered twice by a bandpass filter with an impulse response of $h(t)$. The stage filters the signal and generates the output $Z_{1}(\omega)$ as below:

$$
Z_{1}(\omega)=X(\omega) H(\omega)
$$

where $X(\omega)$ and the $H(\omega)$ are the input signal and the bandpass filter in the frequency domain. The second stage performs a time reversal operation as

$$
Z_{2}(\omega)=Z_{1}^{*}(\omega)=X^{*}(\omega) H^{*}(\omega)
$$

The third stage applies the same bandpass filter again;

$$
Z_{3}(\omega)=Z_{2}(\omega) H(\omega)=X^{*}(\omega) H^{*}(\omega) H(\omega)
$$

which can be simplified as

$$
Z_{3}(\omega)=X^{*}(\omega)|H(\omega)|^{2}
$$

The last and the fourth stage performs a time reversal operation again

$$
Z_{4}(\omega)=Z_{3}^{*}(\omega)=X(\omega)|H(\omega)|^{2}
$$

The overall response of the filter can be represented as

$$
\frac{Z_{4}(\omega)}{X(\omega)}=|H(\omega)|^{2}
$$

where the phase information of the filter is cancelled after the absolute value operation. Therefore this filtering method is referred to as zero-phase filtering. The user must design the filter $h(t)$ while keeping in mind that the input signal $x(t)$ will be filtered twice.
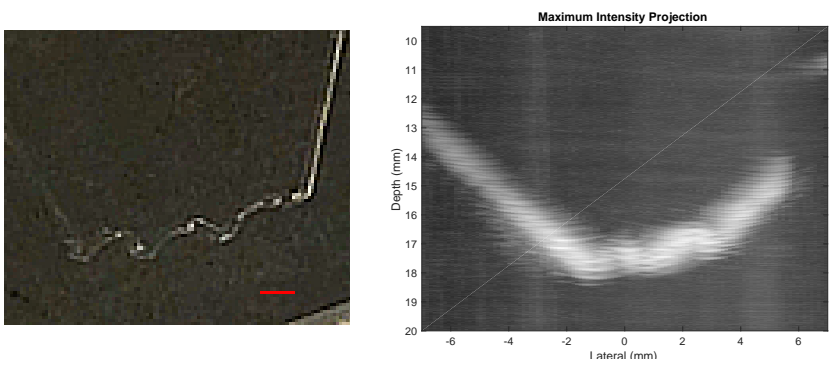

Fig. 1. (Left) Photograph of the test phantom with a $200 \mu \mathrm{m}$ cellulose tube fixed in paraffin gel. A $1 \mathrm{~mm}$ red line is plotted on the image to show the scale. (Right) Maximum intensity projection of 1000 frames used to generate the super-resolution image with a dynamic range of $40 \mathrm{~dB}$.

\section{B. Experimental Setup}

The proposed technique was tested experimentally with a $200 \mu \mathrm{m}$ internal diameter cellulose tube (Hemophan, Membrana) fixed in paraffin gel. Photograph of the test phantom is shown in Fig. 1 (left). A 1:6000 times diluted Sonovue (Bracco S.p.A, Milan, Italy) solution was used during the measurements, which is slightly less than the 1000-2000 dilution ratio that is used typically in clinical scans.

The ULA-OP system (MSD Lab, University of Florence, Italy) was used to acquire data using the LA332 imaging transducer (Esaote, Firenze, Italy) [9]. This is a 144 linear array probe with a $-6 \mathrm{~dB}$ bandwidth ranging from $2-7.5 \mathrm{MHz}$ and a $254 \mu \mathrm{m}$ element pitch. Although it is possible to further improve the image quality and contrast to tissue ratio by using compounding [10], this study only used plane wave imaging. A total of 1000 frames were acquired with a pulse repetition frequency of $100 \mathrm{~Hz}$.

The super-resolution image was generated in three steps. The first stage was a spatio-temporal filtering to remove the tissue echoes from the acquired frames and generate a contrast-mode image [11]. The second stage was zero-phase filtering with a frequency range of $2-7.5 \mathrm{MHz}$ according to the ultrasound probe bandwidth. The last stage was the super-localization of microbubbles, which was performed as explained in [12].

The super-resolution image with frequency decomposition was generated in three steps, where the first and the last stages were the same as above. In the second stage, multiple zerophase bandpass filters were applied to cover the frequency range of $2-5.5 \mathrm{MHz}$ with a step size of $0.5 \mathrm{MHz}$. No microbubble signal was observed above $5.5 \mathrm{MHz}$ for this setup.

The velocity maps were generated by tracking the microbubbles within a three frame temporal window and discarding the false trackings with a maximum velocity threshold. Sizes of the capillary tubes from these images were measured by using the function described in [13].

\section{RESUlTS \& DisCUSSION}

Fig. 2 (top-left) shows the contrast-mode image of two microbubbles inside this capillary flow phantom. Individual microbubbles cannot be localized from this contrast-mode 


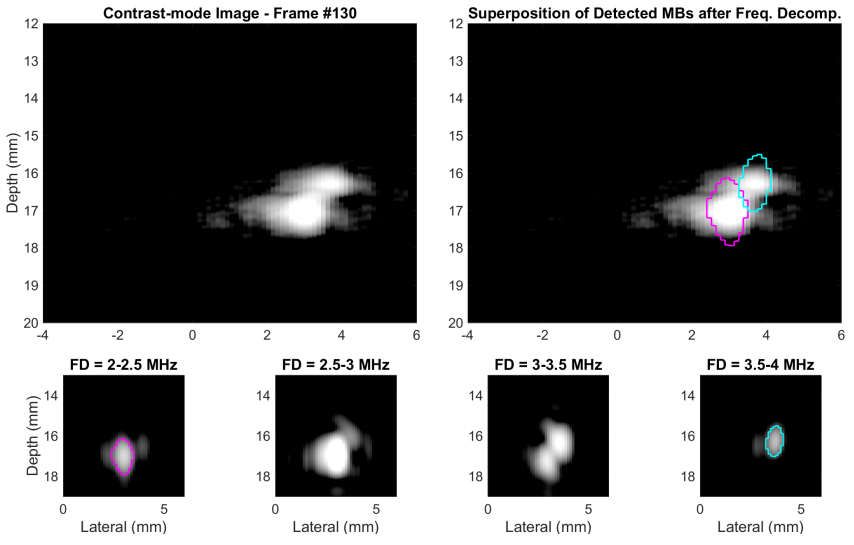

Fig. 2. (Top-left) Contrast-mode image of two microbubbles flowing inside a $200 \mu \mathrm{m}$ tube. (Top-right) Highlights the detected microbubbles after frequency decomposition in different colours overlaid on the contrast-mode image. (Bottom) Contrast-mode images after decomposing the microbubble signal into different frequency decomposition (FD) bands. (Bottom-left) \& (Bottomright) have two different localised microbubbles highlighted with light blue and purple colours.

image, because their point spread functions overlap. The acquired RF signal was decomposed into different components after beamforming as shown in Fig. 2 (bottom). After decomposition, two different microbubbles were detected in Fig. 2 (bottom-left) \& (bottom-right) at frequency bands of $2.0-2.5 \mathrm{MHz}$ and $3.5-4.0 \mathrm{MHz}$ respectively. Fig. 2 (top-right) highlights both detected microbubbles in different colours after frequency decomposition.

After demonstrating the feasibility of the frequency decomposition on a single frame, super-resolution images were generated as shown in Fig. 3 (left). Fig. 3 (top-left) and (bottom-left) are generated by using 250 acquired frames in 2.5 seconds. A total 365 microbubble localisations were achieved from 250 frames while using the frequency decomposition, where the number of localisations were only 129 for the normal case. After processing 1000 frames, a total of 461 microbubbles were localised without frequency decomposition and 1163 with frequency decomposition, where 640 of those were different microbubbles. Approximately 1.4 fold improvement in data acquisition time was achieved with frequency decomposition based on the normalised number of localisations. The localized microbubbles were also tracked to calculate the velocity of the flow inside the tube as shown in Fig. 3 (right).

It is not easy to evaluate the achieved resolution in the super-resolution images due to two factors. First, the located microbubbles are sparse within the tube and it is therefore hard to find the borders from these images, although the structure of the tube is visible. Second, each located microbubble is represented with a smoothed footprint, which had a full width half maximum of $40 \mu \mathrm{m}$. For this reason, the size of the tube is measured from the velocity maps shown in Fig. 3 (right) with an assumption of having laminar flow inside the tube. For laminar flow, the peak velocity is observed at the centre of the tube and it is expected to drop down to $10 \%$ of the peak velocity at a distance of $r=\sqrt{0.9 \times R}$, which is $94.9 \mu \mathrm{m}$ for a tube with radius $R=100 \mu \mathrm{m}$. This means that a velocity profile corresponding to top $90 \%$ of the velocities will be observed in a region with a diameter of $190 \mu \mathrm{m}$ within the $200 \mu \mathrm{m}$ tube. Since each plotted line in the velocity map image has a finite line thickness of $R=20 \mu \mathrm{m}$, the size of the tube is expected to appear as $210 \mu \mathrm{m}$.

The $90 \%$ width of the tube was measured according to this assumption as $125 \mu \mathrm{m}$ and $107 \mu \mathrm{m}$ from the selected regions $\mathrm{A}$ and $\mathrm{B}$ of the velocity map image given in Fig. 3 (top-right). After processing 1000 frames to form the superresolution images (Fig. 3 (middle-right)), the measured size was $140 \mu \mathrm{m}$ and $121 \mu \mathrm{m}$ for the same regions.

For the velocity maps generated by tracking microbubbles after frequency decomposition, the $90 \%$ width of the tube was $171 \mu \mathrm{m}$ and $226 \mu \mathrm{m}$ for the same regions from in Fig. 3 (bottom-right). The size of the tube appears approximately $16 \mu \mathrm{m}$ wider than expected for region $\mathrm{B}$, which might be due to false localizations from partial microbubble signals after using the decomposition method. Although the localisation error is increased after the frequency decomposition, a similar super-resolution image and a velocity map covering most of the capillary tube is achieved from 250 frames in 2.5 seconds. Reducing the total acquisition time is significant for in vivo imaging, since the accumulated tissue motion increases the error in super-resolution images [14].

\section{CONCLUSIONS}

Similar methods based on the detection of single or multiple microbubbles have been previously applied to ultrasound imaging, such as bubble wavelet [15]. Our work shows the feasibility of such a decomposition technique for the first time in super-resolution imaging by detecting two spatially overlapping microbubbles. The ability to separate multiple microbubble echoes is crucial for super-resolution imaging since microbubble concentration is hard to control in vivo.

\section{ACKNOWLEDGMENT}

This work was supported by the Engineering and Physical Sciences Research Council (UK) via grant no. EP/N015487/1 and EP/N014855/1.

\section{REFERENCES}

[1] O. M. Viessmann, R. J. Eckersley, K. Christensen-Jeffries, M. X Tang, and C. Dunsby, "Acoustic super-resolution with ultrasound and microbubbles," Phys. Med. Biol, vol. 58, pp. 6447-6458, 2013.

[2] M. A. O. Reilly and K. Hynynen, "A super-resolution ultrasound method for brain vascular mapping," Medical Physics, vol. 40, no. 110701, 2013.

[3] K. Christensen-Jeffries, R. J. Browning, M.-X. Tang, C. Dunsby, and R. J. Eckersley, "In vivo acoustic super-resolution and super-resolved velocity mapping using microbubbles," IEEE Trans Med Imaging, vol. 34, no. 2, pp. 433-440, 2015.

[4] C. Errico, J. Pierre, S. Pezet, Y. Desailly, O. C. Zsolt Lenkei and, and M. Tanter, "Ultrafast ultrasound localization microscopy for deep superresolution vascular imaging," Nature, vol. 527, pp. 499-507, 2015.

[5] D. Ackermann and G. Schmitz, "Detection and tracking of multiple microbubbles in ultrasound b-mode images," IEEE Trans. Ultrason., Ferroelect., Freq. Control, vol. 63, no. 1, pp. 72-82, 2016. 

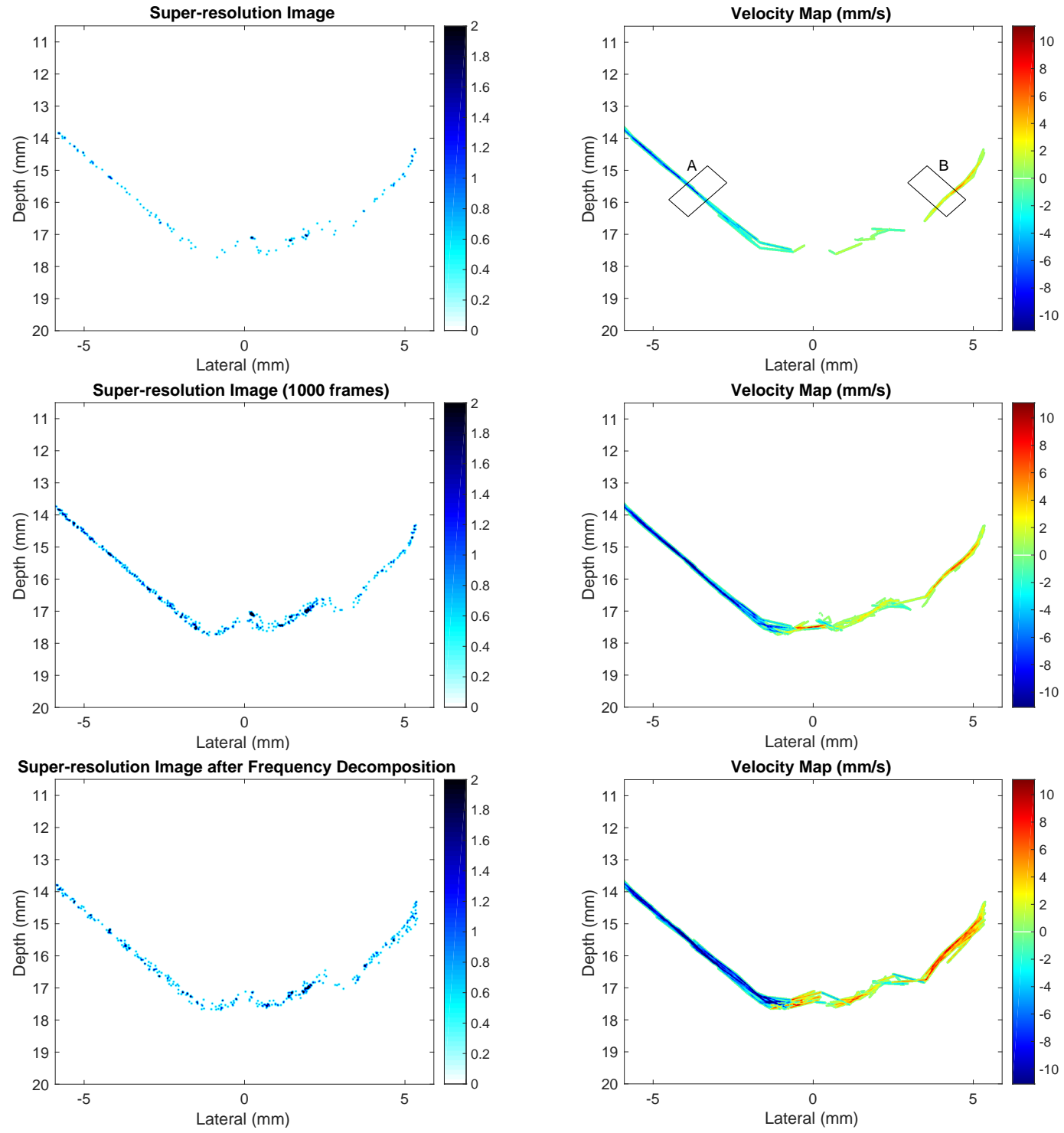

Fig. 3. (Top-left) Super-resolution image of the setup described in Fig. 1 generated from 250 frames. (Middle-left) Super-resolution image of the setup generated from 1000 frames. (Bottom-left) Super-resolution image generated after using the frequency decomposition technique from 250 frames. (Right) The velocity maps of the tracked microbubbles corresponding to figures on the left.

[6] F. Lin, S. E. Shelton, D. Espindola, J. D. Rojas, G. Pinton, and P. A. Dayton, "3-d ultrasound localization microscopy for identifying microvascular morphology features of tumor angiogenesis at a resolution beyond the diffraction limit of conventional ultrasound," Theranostics, vol. 7, no. 1, pp. 196-204, 2017.

[7] Y. Desailly, O. Couture, M. Fink, and M. Tanter, "Sono-activated ultrasound localization microscopy," Applied Physics Letters, vol. 103, no. $174107,2013$.

[8] A. Bar-Zion, C. Tremblay-Darveau, O. Solomon, D. Adam, and Y. C. Eldar, "Fast vascular ultrasound imaging with enhanced spatial resolution and background rejection," IEEE Trans Med Imaging, vol. 36, pp. 169-180, 2017.

[9] E. Boni, L. Bassi, A. Dallai, F. Guidi, V. Meacci, A. Ramalli, S. Ricci, and P. Tortoli, "Ula-op 256: A 256-channel open scanner for development and real-time implementation of new ultrasound methods," IEEE Trans. Ultrason., Ferroelect., Freq. Control, vol. 63, no. 10, pp. 14881495, 2016.

[10] J. Viti, H. J. Vos, N. de Jong, F. Guidi, and P. Tortoli, "Detection of contrast agents: Plane wave versus focused transmission," IEEE Trans. Ultrason., Ferroelect., Freq. Control, vol. 63, no. 2, pp. 203-211, 2016.
[11] C. Demene, T. Deffieux, M. Pernot, B.-F. Osmanski, V. Biran, J.-L. Gennisson, L.-A. Sieu, A. Bergel, S. Franqui, J.-M. Correas, I. Cohen, O. Baud, and M. Tanter, "Spatiotemporal clutter filtering of ultrafast ultrasound data highly increases doppler and fultrasound sensitivity," IEEE Trans Med Imaging, vol. 34, no. 11, pp. 2271-2285, 2015.

[12] K. Christensen-Jeffries, S. Harput, J. Brown, P. N. T. Wells, P. Aljabar, C. Dunsby, M.-X. Tang, and R. J. Eckersley, "Microbubble axial localization errors in ultrasonic super-resolution imaging," IEEE Trans. Ultrason., Ferroelect., Freq. Control, p. in press, 2017.

[13] S. Harput, J. McLaughlan, D. M. Cowell, and S. Freear, "New performance metrics for ultrasound pulse compression systems," in Ultrasonics Symposium (IUS), 2014 IEEE International, 2014, pp. 440-443.

[14] S. Harput, K. Christensen-Jeffries, Y. Li, J. Brown, R. J. Eckersley, C. Dunsby, and M.-X. Tang, "Two stage sub-wavelength motion correction in human microvasculature for ceus imaging," in IEEE International Ultrasonics Symposium (IUS), 2017, pp. 1-4.

[15] D. Wang, Y. Zong, X. Yang, H. Hu, J. Wan, L. Zhang, A. Bouakaz, and M. Wan, "Ultrasound contrast plane wave imaging based on bubble wavelet transform: In vitro and in vivo validations," Ultrasound in Medicine \& Biology, vol. 42, pp. 1584-1597, 2016. 